\title{
A Repository Architecture for Business Process Characterizing Models
}

\author{
Shang Gao and John Krogstie \\ Department of Computer and Information Science (IDI), \\ Norwegian University of Science and Technology (NTNU), Trondheim, Norway \\ shanggao@idi.ntnu.no, krogstie@idi.ntnu.no
}

\begin{abstract}
In this paper, the Business Process Characterizing Model (BPCM) repository architecture is presented. The repository architecture follows a threelayer model composed of a presentation layer, a repository management layer and a storage layer. The objective of the architecture proposed here is to organize BPCM models in a manner that enable their reutilization, and some guidelines on how to use the BPCM repository are provided. Business users are expected to be able to benefit from the advanced functionalities provided by the BPCM repository. Furthermore, based on the evaluation of the BPCM repository by comparing it with some other process repositories, we find that the BPCM repository can offer most features other process repositories are able to provide with the exception of control flow.
\end{abstract}

Keywords: Business Process Characterizing Model, the BPCM Repository, XML.

\section{Introduction}

Today, since organizations are required to react to ever changing market needs in a timely agile manner to stay competitive, an increased flexibility in the business processes is highly desired. Business Process Management (BPM) is the approach to manage the execution of IT-supported business processes from a business stakeholder's point of view rather than from a technical perspective [23].

Information systems development often starts with the development of process models. Model developers usually create process models in a graphical process modeling tool. Subsequently, the process models can be implemented by developers. The process models are supposed to reflect business stakeholders' views on the business domain. However, the proposed processes often deviate from business stakeholders' expectations on the desired processes. One of the main reasons is that some concerns from business stakeholders' views could not be appropriately placed in the process models. And changes which might occur to some tasks in the process models are also not easy to track as the model is transformed among different levels in business systems development according to the needs from various users. In addition, some industry projects and case studies [13], reveal that graphical process 
models are not a good starting point for identifying stakeholder requirements. Many business stakeholders want to start a project with the development of a business oriented model addressing essential business aspects (i.e., what are the essential requirements of the project, what is offered by whom to whom, etc), rather than look at a relative complicated business process model showing how things are executed operationally.

It is believed that modeling the processes of a business domain is a timeconsuming and challenging task. Therefore, it is not surprising that some additional models and frameworks are proposed to capture processes at an abstract level. Also additional frameworks and notations are needed to deal with ad-hoc changes and bridge the gap between business stakeholders and model developers. We have proposed a Business Process Characterizing Modeling language (BPCM) [10] to create a link between business needs and IT implementations. In another paper [27], the authors addressed the problem in the following way: given a high-level process model and a set of previously modeled artifacts in a repository, all semantically annotated, a composition approach should come up with the required set of artifacts from the repository, orchestrating the artifacts in a way that reflects the business process model's structure and business semantics. Compared to some other recent work addressing similar issues, the notable difference in our BPCM work is that we are creating a BPCM language to help business stakeholders create a high level representation of process knowledge in a tabular format in order to share knowledge with other business stakeholders and facilitate the design work of model developers.

During the course of business process support system development, many models in different modeling languages are defined and created. For example, model developers use process models to model business processes. Business stakeholders use some basic modeling tools to express their expectations of the process support system. It is common to have a repository to store all relevant models.

In this paper, we will present a BPCM repository for storing and querying BPCM models. We will also describe the functions that the BPCM repository is able to provide. Thus, the main contributions of this work are the proposed BPCM repository architecture and an evaluation of the BPCM repository by comparing it with other existing process repositories.

An organization can use the BPCM repository for advanced search functionalities. The collection of BPCM models to be analyzed and reused can be retrieved from the BPCM repository. Business users are supposed to profit from the advanced functionalities provided by the repository. For instance, they can search for relevant BPCM models from specific business domains in terms of keywords. Alternatively, they can also perform their searches by using the name of the processes in which they are interested.

The remainder of this paper is organized as follows. Section 2 states the motivation and some related concepts of this research work. In Section 3, we briefly describe the BPCM modeling language, and the BPCM meta-model. Section 4 presents the BPCM repository architecture. Some guidelines on how to use the BPCM repository are provided in Section 5. Section 6 compares the BPCM repository with other process repositories. In Section 7, the contributions of this research are summarized. Finally, Section 8 concludes this work and points out some further research directions. 


\section{Motivation and Related Concepts}

A BPM lifecycle often contains several stages (e.g. Planning, Design, Integration, etc). It is essential to create a fundamental process model in the early phase of a BPM lifecycle, which is a key to the success of business process management of an organization. However, business process modeling is a complicated process. It is time-consuming and error-prone. Therefore, it is important to provide a common repository for business stakeholders to find existing business processes and simplify their effort in the process of business process modeling. We believe that creating a BPCM repository can help them achieve this goal.

Building a good process model is not trivial. It requires a comprehensive understanding of the business domains of the organization, while keeping the business goals, and best practices from other organizations in the same business domains in mind. The constructs of our BPCM model can cover the basic requirements of constructing a business process. Therefore, building a BPCM repository can help business users reuse existing BPCM models for various purposes.

According to Bernstein and Dayal [4], a repository is "a shared database of information about engineered artifacts produced or used by an enterprise". It should provide a common database system for model creation, model modification, model retrieval, model version management. Examples of such artifacts include documents, models, information systems, etc. In our case, the used artifacts in the repository includes: BPCM models on a presentation level, and the BPCM meta-model on a data level. A meta-model is the data that describes meta-data. The concept of meta-model has been successfully used in data interoperability. In [29], the authors proposed the use of meta-models as an advanced architecture for information mediation, where $\mathrm{XML}$ is a main driving force because of its enablement of interoperability. This metamodel based mediation improves the flexibility of choosing common data schema and query language and provides a high level of interoperability. Furthermore, the functionalities for a general repository, as summarized in [4], inspired us to develop repositories that are specific for storing and managing BPCM models.

In general, storing models in a common repository has several benefits. Firstly, it is easy for other users to reuse the relevant models in the future. Second, the models in the repository are subject to common control services. Different access rights can be given to different groups of users by setting up access controls of the repository. Third, it provides a platform for various users to share information so that they can benefit from each other.

Concerning the BPCM model, one of the main benefits of charactering business processes in general is the enablement of searching business oriented process information, such as finding processes in a similar domain or at the same or adjacent step in the SCOR-model (see next section), which can for example later be used for constructing better business process models.

With regard to BPCM models, we use a systematic way to describe BPCM models in order to reuse them. A document file which is specified in XML is defined to describe the characteristics of business processes by following BPCM modeling approach. In an effort to make readers' have an impression on the data level of a BPCM model, a screenshot of a part of a sample BPCM Model in XML is shown in Fig. 1. XML is a marking meta-language that allows the structured representation of 
several types of information. This information can be easily stored and processed on the web. We have seen more and more successful repositories enabled by XML. In our case, XML, which is considered a solution for interoperability, also provides an opportunity to transmit data between BPCM models and other process models. Since most process models can be stored in XML format. For example, BPMN [28] models can be stored in XPDL [8], EPC [2] models can be stored in EPML [20], BPEL [14] can be stored in the BPEL XML format. As a result, this will ease the interaction between BPCM models and other process models. In addition, the repository can also store information about the revision history of various models to better manage them.

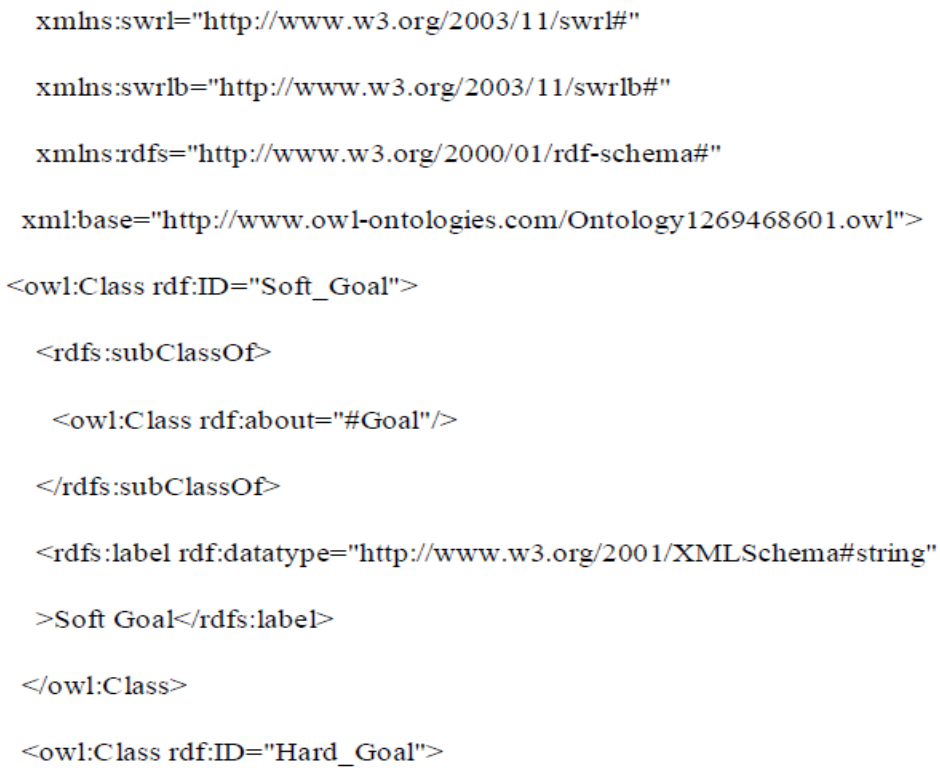

Fig. 1. A part of a sample BPCM Model in XML

\section{Business Process Characterizing Model (BPCM)}

In the course of business process support systems development, model developers often focus on operational and procedural aspects of business process systems, while various business stakeholders are more likely to express different concerns with regard to process models in terms of business oriented concepts. The business process characterizing model (BPCM) is a model used to represent a high level knowledge of business processes.

In an effort to facilitate readers' understanding of the BPCM modeling, we summarize the general definition of the elements for business process characterizing modeling (see Table 1) and present the BPCM metamodel (see Fig. 2) in this section. Since some elements in the BPCM model refer to other ontologies or concepts as presented in the Table 1, a brief description of those concepts and the motivation to incorporte those concepts is provided as follows. 
Concerning the element context, there is no universal or absolute definition for context. [6] describes context as "typically the location, identity, and state of people, groups and computational and physical objects". Context is the reification of the environment, that is, whatever provides a surrounding in which the system operates. People can base their own perceptions or understanding to define context in different ways. In order to better design business process support systems, it is crucial to understand the working contxet and collect and deliver contextual information in a better way. By including a context element in a BPCM model, the correspondent business process support system can be made to serve people better in both mobile and more stationary computing settings.

Table 1. Definition of the elements in BPCM

\begin{tabular}{|l|l|}
\hline \multicolumn{1}{|c|}{ Element } & \multicolumn{1}{c|}{ General Definition } \\
\hline Process & $\begin{array}{l}\text { The business process people want to characterize. This element } \\
\text { can be related to a common business process ontology such as } \\
\text { SCOR [5]. }\end{array}$ \\
\hline Resource & $\begin{array}{l}\text { This element is inspired by the resource concept in the REA } \\
\text { framework [19]. This element can clearly address what are } \\
\text { consumed and what are gained in a business process. }\end{array}$ \\
\hline Actor & $\begin{array}{l}\text { This element describes the people and organizations with } \\
\text { different roles involve in a business process. This element can } \\
\text { illustrate who are important to which business process. }\end{array}$ \\
\hline Context & $\begin{array}{l}\text { It includes contextual characteristics in terms of devices, } \\
\text { software on the devices and networks providing connections } \\
\text { between the devices and others. }\end{array}$ \\
\hline Business Domain & $\begin{array}{l}\text { This element classifies the related business domain(s). We link } \\
\text { this element to the North American Industry Classification } \\
\text { System (NAICS). }\end{array}$ \\
\hline Goal & $\begin{array}{l}\text { This element can address what goals need to be fulfilled in the } \\
\text { business process. The goals may be related to operational goals } \\
\text { and strategical goals. Operational goals are related to hard- } \\
\text { goals, usually covering functional requirements; while strategic } \\
\text { goals are related to soft-goals, which set the basis for non- } \\
\text { functional requirements. }\end{array}$ \\
\hline Process Type & $\begin{array}{l}\text { According to REA [19], REA does not model only exchanges } \\
\text { but also conversions. Exchange and conversion can be seen as } \\
\text { two typical process types. }\end{array}$ \\
\hline This element is designed for keeping track revisions of \\
BPCM models.
\end{tabular}

Recently, W3C has released a draft version of the Delivery Context Ontology (DCO). This ontology constructed in OWL provides a model of characteristics of the environment in which a device interacts with the web or other services. In this research work, we incorporate some key entities of DCO into the context element of BPCM. Some other research work has also started addressing the relationship between context and system development at the requirement level. For instance, [3] 
investigates the relation between context and requirements at the beginning of goal oriented analysis, and [21] extends the application of the problem frames approach with context monitoring and switching problems.

REA [19] [12] was originally conceived as a framework for accounting information systems, but it has subsequently broadened its scope and developed into an enterprise domain ontology [15] and e-commerce framework [1]. The core concepts in the REA ontology are resource, event and agent. The intuition behind this approach is that every business transaction can be described as an event where two agents exchange resources. In order to acquire a resource from other agents, an agent has to give up some of its own resource. The duality of resource transfer is essential in commerce. It never happens that one agent simply gives away a resource to another without expecting another resource back as compensation. Basically, there are two types of events: exchange and conversion [15, 19]. An exchange occurs when an agent receives economic resources from another agent and gives resource back to that agent. A conversion occurs when an agent consumes resources to produce other resources. Annotating process with process type enable users to identify the attribute of business processes around the resource lifecycle. As illustrated in [9], the element resource of BPCM is important in identifying relevant tasks or activities for the construction of process models. For each resource in a BPCM, it should include a message flow which links two associated tasks in a BPMN process model, whereby the source of the message flow connected to the dependee's task and the destination of the message flow connected to the depender's task.

Last but not least, the North American Industry Classification System (NAICS) is a standard for the collection, tabulation, presentation, and analysis of statistical data describing the U.S. economy. NAICS is based on a production-oriented concept, meaning that it groups establishments into industries according to similarity in the processes used to produce goods or services. Each business process is labeled with a business domain. This is of help for model users to search or retrieve business processes within specific business domain.

The BPCM meta-model [11] is described in Fig. 2, which presents the major modeling concepts and the relationships between them using a UML class diagram.

The central class of the meta-model is process. One process may consists of zero or more sub process(es). All other main classess can be associated with the central class process. Around the central class, business stakeholders could express different concerns, like required actors, required resources, contextual information, and so on. We use UML generalizations in case of elements extensions (e.g. context, process types). Then, process model developers can take these concerns into consideration when designing process models accordingly.

The delivery context ontology mainly focuses on the following three entities: a). environment including information about the location and network, b). software describes whether the delivery context supports certain APIs, document formats, operating systems, etc, c). Hardware provides information about various hardware capabilities including display, input, memory, etc. For the class context, we do not attempt to cover all entities above. We focus on two major aspects: location and network information. For example, a user using its device is connected to an information system through a wireless network or wired network. Mobile workers need to work in various locations (e.g. at home, in the office, on the way). Mobility 
has become an important trend for various activities in both working and non-working settings. Several taxonomies of remote mobility are discussed in the literature. In [16], the authors distinguish between travelling, visiting and wandering. Travelling is movement between different locations in a vehicle. Visiting is a prolonged period spent in one location before moving back to the original location or on to another one. Wandering is moving about — usually on foot — in the local area.

Business process scenario itself is a complex, dynamic network that involves many business stakeholders. Integrating SCOR with the BPCM meta-model for the business process development process will facilitate knowledge sharing and communication among the various parties involved in the processes. As illustrated in Fig. 2, we have incorporated process best practices and process levels from SCOR into the BPCM meta-model.

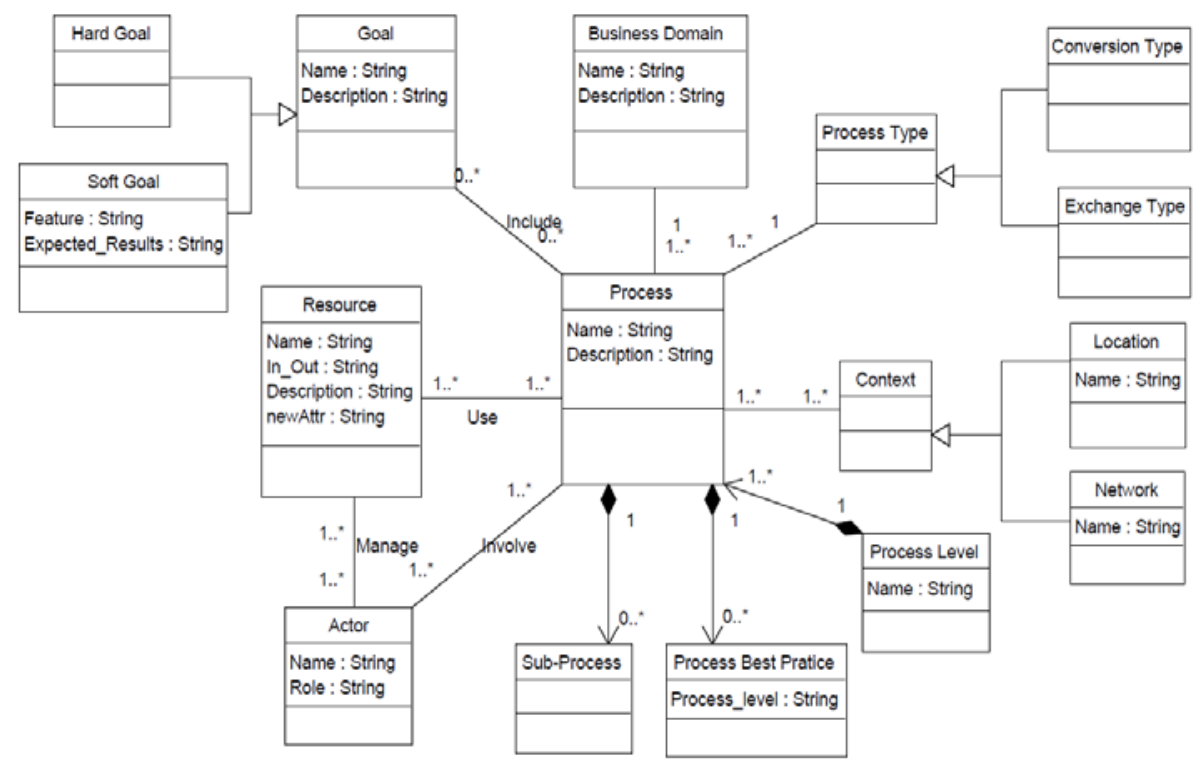

Fig. 2. BPCM Meta Model

\section{The BPCM Repository Architecture}

This section presents the architecture for the BPCM repository. The architecture, as shown in Fig. 3, follows a three-layer model composed of a presentation layer, a repository management layer and a storage layer. Moreover, the BPCM repository architecture can link to some external systems.

The objective of the architecture proposed here is to organize BPCM models in a good manner to enable their reutilizations. Because of the reusing and sharing facilities of the repository, it is possible for various business users to use the existing knowledge of different business processes, which have already been built by other relevant organizations. 


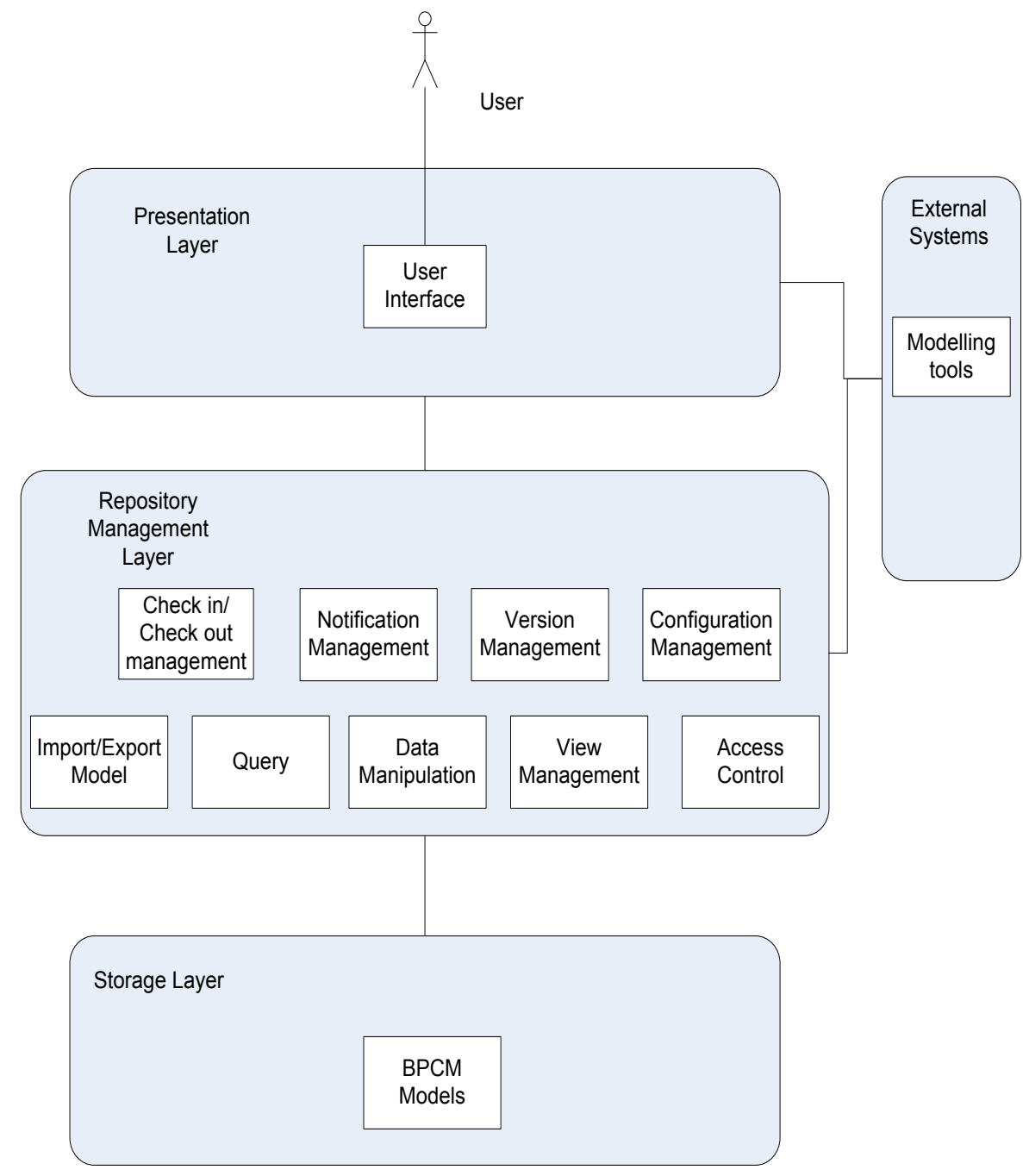

Fig. 3. The BPCM Repository Architecture

The presentation layer provides a graphic user interface for users to interact with the BPCM repository. As a result, the users can easily interact with the various functions provided by the repository. This layer can also allow users to browse and navigate the repository easily, give queries to search for interesting data or BPCM models, and visualize the results in some given formats according to their preferences. For example, given some key words such as a specific business domain, all the related BPCM models in this domain, already existed in the repository, will appear on the user interface. Then, users can review the retrieved BPCM models for various usages.

The repository management layer provides the general amenities of a repository, such as access control, notification management, querying, view management, version 
management, and check-in/check-out management, etc. The access control amenity can ensure the right people have the right access to the right objects and data in the repository. Notification management enables notifications to be generated in case any change occurs to any object in the repository. Querying functionality enables users to search for the desired information in the repository. Version management allows users to keep track of changes occurred to the specific object in the repository. It makes it possible to maintain multiple BPCM models of the same business process. The view management provides a possibility for users to have multiple views on a BPCM model. Check in/Check out allows users to check in data/objects to the repository or check out data/objects from the repository. For example, a user can obtain a copy of the BPCM model for a specific business process analysis and design from the BPCM repository and store it at a local directory (Check out). A user can also view an extracted BPCM model and make some modifications to the model. Once all the changes are made to the model, the user can export it back to the repository (Check in). It is possible that more than one user are working on the same BPCM model and may be modifying the model at the same time. Thus, it is necessary to offer a solution to deal with it. One possible solution is to simply lock the current model, so that other users cannot make changes on the current version of the model until it is checked back into the repository by releasing the lock.

The storage layer stores the BPCM models in terms of XML formats. This layer aims to provide faster querying services and better navigation services of the BPCM repository. The data structure of the BPCM models in this layer is described in the BPCM meta-model (see Fig. 2). Thanks to the power of meta-model, all the BPCM models are transferable between a tabular format and XML in this layer. Users can add new BPCM models to the BPCM repository. Then, the BPCM models will be stored in the storage layer. From that time onwards, these BPCM models can be accessed by all registered users of the BPCM repository. This layer is mainly invoked by the repository management layer.

In addition, the presentation layer and repository management layer can be made to also interact with external systems (e.g. process modeling tool). For example, technical users can open a process modeling tool (e.g., BPMN modeling tools) from the graphical user interface at the presentation layer of the BPCM repository to model relevant business processes based on the approved BPCM models by business stakeholders. And external systems can invoke the data and objects in the BPCM repository by interacting with the repository management layer.

\section{Guidelines to Use the BPCM Repository}

In this section, we provide some guidelines for business users to use the BPCM repository. Prior to providing the business requirements to model developers to develop a business process support system, the BPCM repository should be queried by business stakeholders to ensure that the useful best practices and relevant information from the existing BPCM models, which are appropriate to model a process support system from business perspective, has been taken into consideration. 


\subsection{Identify the Appropriate Candidate BPCM Models}

The identification phase aims at identifying potential reusable BPCM models. Some relevant BPCM models are supposed to be extracted from the BPCM repository based on a given search keyword. To limit the retrieved models to a specific business domain, one or more keywords can be used to search for the most relevant BPCM models. This would reduce analysis complexity and time effort of this identification process. Then, the retrieved BPCM models can be assessed according to business stakeholders' needs to select the best BPCM model to be used.

\subsection{Redesign the BPCM Model/ Create New BPCM Model}

The result of the query against the BPCM repository may be a full match, a partial match, or no match. A full match indicates that the extracted BPCM model is able to provide all business oriented information the business stakeholder expects. In this case, the BPCM model can be entirely reused as a sketch model for business process support system development. But in most cases, the extracted BPCM model may need to be redesigned to better fulfill the needs of business stakeholders or achieve business goals. For example, users may add or delete some attributes to some elements of the BPCM model according to their requirements on the business process. When some changes are made to BPCM models, it is better to annotate the BPCM models with additional information in terms of the version element of the BPCM modeling language. This will help users explore the revision history information on a specific BPCM model more efficiently and effectively for later use. In case no relevant BPCM models could be found from the BPCM repository, the graphical user interface at the presentation layer will allow users to model a completely new BPCM model from sketch, so that other business users may benefit from it in the future.

\section{Comparing BPCM Repository with Other Existing Repositories}

In this section, some of existing process repositories are presented firstly. Then, we evaluate the similarities and differences between the BPCM repository and the other process repositories.

\subsection{Existing Process Repositories}

\section{MIT process handbook}

The MIT process handbook [18] is a knowledge base of process description. This handbook focuses on organizing knowledge about processes rather than providing detailed process models. The knowledge about the process is provided in a text-based manner. The goal of the MIT process handbook is to develop rich online libraries for sharing and managing many kinds of knowledge about business. A process may be a generalization/specialization of another process, and a process may have other processes as parts or use other processes. Processes available in the repository are grouped into ten root categories: procurement, supply chain management, marketing, sales, information systems, human resources, strategic planning, finance/accounting, manufacturing/ logistics, and engineering. 


\section{RepoX}

Repox [24] is an XML based process model repository, that has been developed for the METEOR workflow system. It maps XML documents to a relational-object database and also provides extraction/retrieval, version control, check in/check out, and querying functions. It stores the control flow aspect of process models along with the data that is used in the processes. And it is mainly designed as the repository for a workflow engine. In addition, it supports for adaptive workflows, which may need workflow definition information from the repository at runtime, in a dynamically changing environment.

\section{The BPMN repository}

The BPMN repository architecture [25] is a conceptual architecture for crossenterprise processes planning, implementation, and controlling. Core component of the architecture is a distributed repository managing all required data and information, which especially obtains a process-oriented view on collaboration networks. Cross organizational processes need close coordination among networking partners. This is achieved through the integration of business process models. The architecture prescribes that processes and related information should be stored in XML formats.

\section{The Semantic business process repository}

The Semantic Business Process Repository (SBPR) [17] is an ontology-based repository for storing business process models. It is used for storage and management of business process modeling artifacts. This repository is open for change (e.g. updating, deletion) by potential users. It requires that the repository is configured with a process ontology. The ontologies must be specified in WSML. It supports querying, versioning and check in /check out.

In SBPR, business process models are enriched by annotating business process artifacts with entities from pre-defined ontologies such as organizational ontologies, Semantic Web Service ontology, business functions ontology, resource ontology, and domain ontologies. It stores instances of process models which are based on ontologies.

\section{SCOR}

Supply-Chain Operations Reference Model [5] can be seen as a repository that stores business process related to supply chain management. SCOR is a process reference model designed for effective communications among supply chain partners. SCOR integrates concepts of business processes, benchmarking, and best practices into a cross-functional framework. From a business process reengineering perspective, the SCOR model builds a hierarchy of supply chain processes, which can be divided into three levels of details [5]: process type, process category, and process element. SCOR classifies the operations of supply chain as Plan, Source, Make, Deliver and Return.

\section{BPEL Repository}

The BPEL Repository [26] is an Eclipse plug-in originally built for BPEL business processes and other related XML data. This repository uses the XML format as its external format and stores BPEL models as objects in an EMF repository. 
The repository can easily be extended with additional XML schemas because of its flexible architecture. Users can query the XML files as EMF objects using an objectoriented query language, namely the Object Constraint Language (OCL) that is part of the UML specification.

\subsection{Comparison with Other Process Repositories}

In order to compare the BPCM repository with other repositories that were presented in the last section, a number of criteria are identified. The criteria were selected by surveying the literature on requirements for process repositories [7, 22]. The resulting criteria are presented in the column one in the Table 2.

Table 2. Repositories Comparison

\begin{tabular}{|l|l|l|l|l|l|l|l|}
\hline & $\begin{array}{l}\text { BPCM } \\
\text { Repository }\end{array}$ & $\begin{array}{l}\text { MIT } \\
\text { Process } \\
\text { Handbook }\end{array}$ & RepoX & $\begin{array}{l}\text { BPMN } \\
\text { Repository }\end{array}$ & SBPR & SCOR & $\begin{array}{l}\text { BPEL } \\
\text { Repository }\end{array}$ \\
\hline Control Flow & Not Support & $\begin{array}{l}\text { Not } \\
\text { Support }\end{array}$ & Support & Support & Support & Support & Support \\
\hline Retrieval & Search & Search & Xquery & $\begin{array}{l}\text { Not } \\
\text { Available }\end{array}$ & WSML & Search & OCL \\
\hline Model Storage & XML & Textual & XML & XML & XML & Textual & XML \\
\hline $\begin{array}{l}\text { Integration } \\
\text { with other } \\
\text { Tools }\end{array}$ & Yes & No & No & Yes & Yes & No & Yes \\
\hline $\begin{array}{l}\text { Relation with } \\
\text { Model Building }\end{array}$ & $\begin{array}{l}\text { Provide } \\
\text { Process } \\
\text { related Info }\end{array}$ & $\begin{array}{l}\text { Provide } \\
\text { Process } \\
\text { related Info }\end{array}$ & $\begin{array}{l}\text { Reuse/ } \\
\text { Adapt }\end{array}$ & $\begin{array}{l}\text { Reuse/ } \\
\text { Adapt }\end{array}$ & $\begin{array}{l}\text { Reuse/ } \\
\text { Adapt }\end{array}$ & $\begin{array}{l}\text { Provide } \\
\text { Process } \\
\text { related } \\
\text { Info }\end{array}$ & $\begin{array}{l}\text { Reuse/ } \\
\text { Adapt }\end{array}$ \\
\hline $\begin{array}{l}\text { Goal Inclusion } \\
\text { Yes }\end{array}$ & No & No & No & No & Partly & No \\
\hline $\begin{array}{l}\text { Domain } \\
\text { Specific }\end{array}$ & No & No & No & No & No & Yes & No \\
\hline $\begin{array}{l}\text { Version } \\
\text { Management }\end{array}$ & Yes & No & Yes & No & Yes & No & No \\
\hline
\end{tabular}

The BPCM repository and the MIT process handbook do not consider storage of control-flow aspect of process models. More specifically, the BPCM repository focuses on storing tabular descriptions of major characteristic of business processes in XML files, whereas the MIT process handbook aims at storing textual descriptions of the processes and the activities that occur within those processes.

Generally, the repositories can be used in two different ways. In the first category, the extracted models and data can be used as knowledge based foundation to guide developers to model process models. The MIT process handbook, SCOR, and the BPCM repository fall into this category. The second way focuses on reuse of the existing process models/workflows in the repository to develop customized process models. This category includes BPMN repository, BPEL repository and RepoX. 
Most repositories use XML based formats for data interchange. The two exceptions are the MIT process handbook, and SCOR. The MIT process handbook is available only through a web-based user interface, through which users interact using natural languages. SCOR is also a web-based textual description resource. Most repositories are able to provide search or query functions in terms of different query languages or search methods.

The BPCM repository, the MIT process handbook, and SCOR are mainly intended to store process related information rather than implementation details of business process models.

All these repositories are mainly meant to store process models and process related information. Most of the repositories in the current formats do not support the integration with external tools. Therefore, we believe that it is beneficial to integrate those repositories with some external modeling tools. That is why we would like to connect the BPCM repository with other external tools.

Concerning the goal inclusion, this is another aspect our BPCM repository distinguishes from the other repositories. Although SCOR includes process metrics, this is only one type of relevant goals to be represented. Goal inclusion means that business goals are given with the process. The BPCM modeling approach allows business users to depict the business goals a process aims to achieve, which in turn the BPCM repository can benefit from.

Only one of the repositories is limited in terms of business scope. SCOR can only store process related information about supply chain management.

The BPCM repository, RepoX, and SBPR can provide facilities for version management.

\section{Contributions}

Since business process modeling is growing in importance and popularity in most organizations nowadays, business process knowledge storing, sharing and reusing in terms of process repositories are becoming more and more important. In this study, we strive to achieve a shared database of process knowledge through the BPCM repository. The main contribution of this research is the three-layer BPCM repository architecture, including a presentation layer, a repository management layer and a storage layer. Another contribution of this research is the evaluation on the similarities and differences between the BPCM repository and other existing process repositories. This understanding is important to the success of process repositories endeavors and efforts.

Another possible implication that can be drawn from this research is that the BPCM models in the BPCM repository could be used to help design and deploy process models that better match an organization's goals from a business point of view. Since the data for BPCM models could be represented in XML, BPCM models are able to provide a higher level of interoperability with other process models. We hope the insights from this research can draw practitioners' attention to create a better process repository by including the essential characteristics of business processes to facilitate communications between business stakeholders and model developers. 


\section{Conclusion and Future Work}

In this paper, the BPCM repository architecture is presented. It follows a three-layer model composed of a presentation layer, a repository management layer and a storage layer. The BPCM repository architecture is the foundation to provide functions, such as access, import/export, and so on, to manage all major characteristic of business processes of enterprises in terms of BPCM models in the form of XML documents. Since the BPCM meta-model is of help to transfer BPCM models from a tabular format to a XML file, this repository can also be regarded as an XML repository.

Based on the evaluation of the BPCM repository by comparing with some other process repositories, we find that the BPCM repository can offer most features that other process repositories are able to provide with the exception of control flow. This is partially attributed to the fact that BPCM models, which mainly focus on providing a simple view of a business process in terms of characteristics, differ from business process models, which are more about how a business scenario is implemented in terms of executable business process models. We believe this kind of modeling approach is what most business stakeholders expect.

However, it must be admitted that the evaluation of the BPCM repository is currently quite limited since we have not tested it in some case studies. Future research will implement the functions of the BPCM repository and test the usability of the BPCM repository in some business cases. More specifically, we are currently evaluating the usability of the BPCM modeling language in a real conference arangement case. Following an explanation of the BPCM modeling language, how to use the language, and the tabular template to represent a process, participants are asked to characterize the business processes in connection to a secientfic conference series.

\section{References}

1. UN/CEFACT Modeling Methodology (UMM) User Guide (2007)

2. Aalst, W.v.d.: Formalization and Verification of Event-driven Process Chains. Information and Software Technology 41(10), 639-650 (1999)

3. Ali, R., Dalpiaz, F., Giorgini, P. (eds.): A Goal Modeling Framework for Selfcontextualizable Software, LNBIP, vol. 29, pp. 326-338. Springer, Heidelberg (2009)

4. Bernstein, P.A., Dayal, U.: An Overview of Repository Technology. In: Proceedings of the 20th International Conference on Very Large Data Bases, Morgan Kaufmann Publishers Inc., Santiago de Chile (1994)

5. Council, S.-c. SCOR Model 8.0 Quick Reference Guide (2006)

6. Dey, A.K., Abowd, G.D., Salber, D.: A conceptual framework and a toolkit for supporting the rapid prototyping of context-aware applications. Human-Computer Interaction 16(2), 97-166 (2001)

7. Feldmann, R.L. and Nick, M.: Guidelines for Evaluation and Improvement of Reuse and Experience Repository Systems through Measurement Programs. In: The Third European Software Measurement Conference (2000)

8. Fischer, L.: Workflow handbook 2005. Workflow Management Coalition, WfMC (2005) 
9. Gao, S., Krogstie, J.: A Combined Framework for Development of Business Process Support Systems. In: Persson, A., Stirna, J. (eds.) The Practice of Enterprise Modeling, vol. 39, pp. 115-129. Springer, Heidelberg (2009)

10. Gao, S., Krogstie, J.: Facilitating Business Process Development via a Process Characterizing Model. In: International Symposium on Knowledge Acquisition and Modeling 2008. IEEE CS Los Alamitos (2008)

11. Gao, S., Krogstie, J.: A Meta-model for a Language for Business Process Characterizing Modelling. In: Popplewell, K., Harding, J., Poler, R., et al. (eds.) Enterprise Interoperability IV, pp. 69-79. Springer, London (2010)

12. Geerts, G.L., McCarthy, W.E.: An Accounting Object Infrastructure for Knowledge-Based Enterprise Models. IEEE Intelligent Systems 14(4), 89-94 (1999)

13. Gordijn, J., Akkermans, H., Vliet, H.V.: Value Based Requirements Creation for Electronic Commerce Applications. In: HICSS 2000. IEEE CS, Los Alamitos (2000)

14. Havey, M.: Essential Business Process Modeling. O’Reilly Media, CA (2005)

15. Hruby, P.: Model-Driven Design Using Business Patterns. Springer, New York (2006)

16. Kristoffersen, S., Ljungberg, F.: Mobility: From stationary to mobile work. In: Braa, K., Sørensen, C., Dahlbom, B. (eds.) Planet Internet (2000)

17. Ma, Z., Wetzstein, B., Anicic, D., et al.: Semantic Business Process Repository. In: The Workshop on Semantic Business Process and Product Lifecycle Managemen 2007. CEUR-WS (2007)

18. Malone, T.W., Crowston, K., Herman, G.A.: Organizing Business Knowledge: The MIT Process Handbook. MIT Press, Cambridge (2003)

19. McCarthy, W.E.: The REA accounting model: a generalized framework for accounting systems in a shared data environment 57, 554-578 (1982)

20. Mendling, J., Nüttgens, M.: EPC markup language (EPML): an XML-based interchange format for event-driven process chains (EPC). Information Systems and E-Business Management 4(3), 245-263 (2006)

21. Salifu, M., Yu, Y., Nuseibeh, B.: Specifying Monitoring and Switching Problems in Context. In: RE 2007. IEEE CS Press, Los Alamitos (2007)

22. Shahzad, K., Andersson, B., Bergholtz, M., et al. (eds.): Elicitation of Requirements for a Business Process Model Repository, pp. 44-55 (2009)

23. Smith, H., Fingar, P.: Business Process Management: The Third Wave. Meghan-Kiffer Press (2003)

24. Song, M., Miller, J., Arpinar, I.: RepoX: An XML Repository for Workflow Design and Specifications. Univeristy of Georgia, USA (2001)

25. Theling, T., Zwicker, J., Loos, P., et al. (eds.): An Architecture for Collaborative Scenarios Applying a Common BPMN-Repository, pp. 169-180 (2005)

26. Vanhatalo, J., Koehler, J., Leymann, F.: Repository for Business Processes and Arbitrary Associated Metadata. In: Demo section at BPM 2006 (2006)

27. Weber, I., Markovic, I., Drumm, C.: A Conceptual Framework for Composition in Business Process Management, pp. 54-66 (2007)

28. White, S.A.: Introduction to BPMN (2005)

29. Zhao, L., Siau, K.: Information Mediation Using Metamodels: An Approach Using XML and Common Warehouse Metamodel. Journal of Database Management 18(3), 69-82 (2007) 